\title{
STREAMING MOTIONS TOWARD THE SUPERMASSIVE BLACK HOLE IN NGC 1097
}

\author{
Kambiz Fathi, ${ }^{1}$ Thaisa Storchi-Bergmann, ${ }^{2}$ Rogemar A. Riffel, ${ }^{2}$ Claudia Winge, ${ }^{3}$ David J. Axon, ${ }^{1}$ \\ Andrew Robinson, ${ }^{1}$ Alessandro Capetti, ${ }^{4}$ And Alessandro Marconi ${ }^{5}$ \\ Received 2005 November 22; accepted 2006 March 3; published 2006 March 22
}

\begin{abstract}
We have used GMOS-IFU and high-resolution HST-ACS observations to map, in unprecedented detail, the gas velocity field and structure within the $0.7 \mathrm{kpc}$ circumnuclear ring of the SBb LINER/Seyfert 1 galaxy NGC 1097. We find clear evidence of radial streaming motions associated with spiral structures leading to the unresolved $(<3.5 \mathrm{pc})$ nucleus, which we interpret as part of the fueling chain by which gas is transported to the nuclear starburst and supermassive black hole.
\end{abstract}

Subject headings: galaxies: active — galaxies: kinematics and dynamics — galaxies: nuclei

Online material: color figure

\section{INTRODUCTION}

Mechanisms of mass transfer in active galaxies from galactic scales down to nuclear scales have been the subject of many theoretical and observational studies (e.g., Shlosman et al. 1990; Emsellem et al. 2003; Maciejewski 2004; Knapen 2005). Simulations have shown that nonaxisymmetric potentials efficiently promote gas inflow toward the inner kiloparsec-scale regions (e.g., Englmaier \& Shlosman 2004). Furthermore, recent observations have revealed that structures such as small-scale disks or nuclear bars and associated spiral arms are frequently observed in the inner regions of active galaxies, suggesting that they could be the means by which gas is transported from the kiloparsec scale down to within a few tens of parsecs of the active nucleus (e.g., Knapen et al. 2000; Emsellem et al. 2001; Maciejewski et al. 2002; Crenshaw et al. 2003; Fathi et al. 2005).

The nearby (14.5 Mpc) LINER/Seyfert 1 galaxy NGC 1097 is an important "laboratory" in this context. It hosts a largescale bar as well as a secondary bar within its $0.7 \mathrm{kpc}$ circumnuclear ring (e.g., Shaw et al. 1993). Since the discovery of its double-peaked $\mathrm{H} \alpha$ emission lines (Storchi-Bergmann et al. 1993), there have been several studies following the "fate" of the gas accumulated in the center (e.g., Storchi-Bergmann et al. 2003).

Recently, Prieto et al. (2005, hereafter PMR05) have presented high-resolution ground-based near-infrared images obtained with the Very Large Telescope. They reported the discovery of spiral structures within the inner 300 pc of the galaxy, which can be traced to within $\approx 10$ pc of the nucleus. PMR05 argued that these spirals trace cool dust and that they could be channels by which cold gas and dust flow toward the nuclear supermassive black hole (SMBH).

Here we present two-dimensional maps of the gas kinematics and structure within the inner $0.5 \times 1.0 \mathrm{kpc}$ of NGC 1097 , which appear to trace the fueling path to the unresolved nucleus.

\footnotetext{
${ }^{1}$ Physics Department, Rochester Institute of Technology, 85 Lomb Memorial Drive, Rochester, NY 14623; kambiz@cis.rit.edu, djasps@rit.edu, axrsps@rit.edu.

${ }^{2}$ Instituto de Fìsica, Universidade Federal do Rio Grande do Sul, Av. Bento Goncalves 9500, 91501-970 Porto Alegre RS, Brazil; thaisa@ufrgs.br, rogemar@ufrgs.br.

${ }^{3}$ Gemini Observatory, Casilla 603, La Serena, Chile; cwinge@ gemini.edu.

${ }^{4}$ INAF-Osservatorio Astronomico di Torino, Strada Osservatorio 20, 10025 Pino Torinese, Italy; capetti@to.astro.it.

${ }^{5}$ INAF-Osservatorio Astrofisico di Arcetri, Largo Fermi 5, I-50125 Florence, Italy; marconi@arcetri.astro.it.
}

Our study is based on high signal-to-noise ratio $(\mathrm{S} / \mathrm{N})$ spectra obtained with the integral-field unit (IFU) of the Gemini MultiObject Spectrograph at Gemini South (GMOS-S); these spectra reveal the inner gas kinematics in unprecedented detail. We use these data in combination with high-resolution images obtained with the Advanced Camera for Surveys (ACS) of the Hubble Space Telescope (HST) to cross-correlate the gas kinematics with the morphology of the nuclear spiral structure.

\section{OBSERVATIONS}

Our two-dimensional spectroscopic observations of NGC 1097 were obtained with the GMOS IFU on the Gemini South Telescope, on 2005 January 8 (GS-2004B-Q-25, PI: T. StorchiBergmann), using the R400_G5325 grating and r_G0326 filter. These spectra cover the wavelength range 5600-7000 $\AA$ at a spectral resolution of $R \approx 3500$ ( $85 \mathrm{~km} \mathrm{~s}^{-1}$ FWHM). We observed three consecutive fields of angular dimensions $5^{\prime \prime} \times 7^{\prime \prime}$ each, covering in total $15^{\prime \prime}$ along the major axis of the galaxy (position angle P.A. $=129^{\circ}$ ) and $7^{\prime \prime}$ along the minor axis, sampled by hexagonal lenslets with projected diameters of 0 " 2 . Three exposures of $600 \mathrm{~s}$ were obtained for each field, slightly shifted along P.A. $=40^{\circ}$ in order to correct for detector defects. The spectra were reduced using standard tasks in IRAF. The products of the reductions are three wavelength and flux-calibrated data cubes, resampled to rectangular arrays of $50 \times 70$ spectra, each corresponding to an angular coverage of $00^{\prime \prime} 1 \times 00^{\prime \prime} .1$.

The HST-ACS image for NGC 1097 was obtained (GO 9782; PI: D. J. Axon) using the High Resolution Camera mode (0"025 pixel $^{-1}$ and a field of view of $26^{\prime \prime} \times 29^{\prime \prime}$ ), through the FR656N filter. The ACS image was reduced and flux-calibrated using the standard CALACS pipeline.

\section{RESULTS}

\subsection{Spectroscopy}

We used the two-dimensional spectroscopic observations to map the kinematics of the ionized gas in the central $0.5 \times 1 \mathrm{kpc}$. Inspection of the individual spectra reveals the presence of the [O I] $\lambda \lambda 6300,6364, \mathrm{H} \alpha \lambda 6563,[\mathrm{~N}$ II $] \lambda \lambda 6548,6583$, and [S II] $\lambda \lambda 6717,6731$ emission lines throughout most of the observed field. The velocity field was derived from the central wavelengths of unconstrained Gaussian profiles fitted to the narrow $\mathrm{H} \alpha$ component and to the $[\mathrm{N}$ II] emission lines, as these lines have good $\mathrm{S} / \mathrm{N}$ throughout the entire field. Within the inner 0".5, we introduced 
three additional Gaussian components to fit and remove the underlying double-peaked broad component of the $\mathrm{H} \alpha$ line (see Fig. 1). However, this does not significantly affect the velocity field derived from the narrow lines. Since the velocity fields of $\mathrm{H} \alpha$ and $[\mathrm{N}$ II] $\lambda 6583$ are identical within the uncertainties $(\approx 10$ $\mathrm{km} \mathrm{s}^{-1}$ ), we chose the latter to represent the gaseous velocity field of the galaxy because of its higher $\mathrm{S} / \mathrm{N}$.

In the top panels of Figure 2, we present the two-dimensional maps of the total flux in the bandpass, the integrated [N II] $\lambda 6583$ flux, and the velocity dispersion $(\sigma)$. The nucleus is at position $(0,0)$, while a part of the circumnuclear $0.7 \mathrm{kpc}$ starforming ring is visible at the top of the panels. The $\sigma$ map (corrected for the instrumental resolution) has circumnuclear values of around $40 \mathrm{~km} \mathrm{~s}^{-1}$ and decreases outward to values of $\approx 15 \mathrm{~km} \mathrm{~s}^{-1}$, as one approaches the circumnuclear ring at about 7 " southeast from the nucleus.

The velocity field is shown in the bottom left panel of Figure 2 . It is clearly dominated by rotation, with distortions indicative of significant noncircular motions. Similar distortions have previously been observed in the large-scale gas kinematics of nearby disk galaxies (e.g., Visser 1980; Zurita et al. 2004; Emsellem et al. 2006), where they were found to trace the galaxy spiral arms. In order to try to isolate these noncircular motions, we have fitted an exponential thin disk model (Freeman 1970) to the velocity field using a method developed by Fathi (2004). In the bottom middle panel of Figure 2, we illustrate the best model, which has a deprojected maximum amplitude of $\approx 200 \mathrm{~km} \mathrm{~s}^{-1}$, a disk scale length of 3.5 , a kinematic P.A. of $137^{\circ}$, and a disk inclination of $35^{\circ}$. This inclination is similar to that derived by Storchi-Bergmann et al. (2003) for the nuclear accretion disk but is smaller than the value of $46^{\circ}$ found from the large-scale gas kinematics by Storchi-Bergmann et al. (1996). The kinematic P.A. agrees with the value of $135^{\circ}$ from Storchi-Bergmann et al. (1996) and Emsellem et al. (2001). We have tested the robustness of the model against extinction variations across the field by repeating the fit several times, masking out the regions most affected by the distortions. We found no significant variations in the model parameters.

We constructed a residual map by removing the disk model from the observed velocity field. The result is shown in the bottom right panel of Figure 2, where it can be observed that the residuals display amplitudes of $\approx 50 \mathrm{~km} \mathrm{~s}^{-1}$. Moreover, the velocity residuals are distributed in a spiral pattern, centered on the nucleus. We identify three main arms, designated 1, 2, and 3, which are outlined by white dots superposed on the residual map. The residuals indicate redshifts along arm 2 but blueshifts along arms 1 and 3. As arms 1 and 2 are oriented approximately along the minor axis, and the southwest is the near side of the galaxy (Storchi-Bergmann et al. 1996), we conclude that the residuals indicate radial inflow of gas along these circumnuclear arms. This follows from the geometry of a configuration where the radial motions occur along the spiral arms that are in the plane of the disk. In this case, one expects the maximum line-of-sight velocities along the minor axis. Furthermore, this implies that the spiraling direction is clockwise. This simple setup does not fully explain the observed blueshift along the inner parts of arm 3, where outflows or individual gas clouds could complicate the observed velocities.

\subsection{Imaging}

We used two different techniques of image analysis to investigate the structures present in the image. The first is gen-

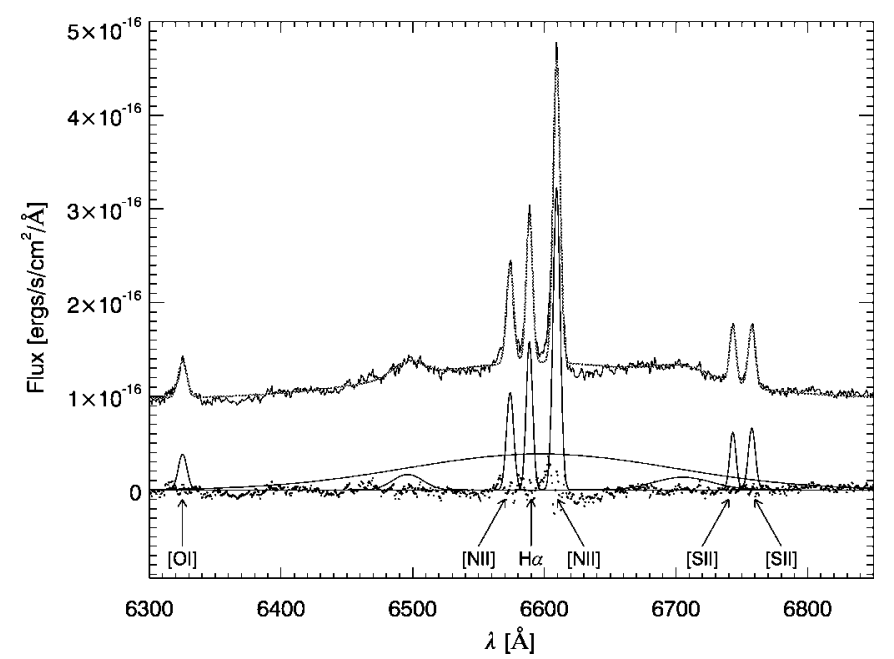

FIG. 1.-Sample GMOS spectrum of the nucleus illustrating the multi-Gaussian fit used to determine the velocities of the narrow lines and remove the underlying double-peaked broad $\mathrm{H} \alpha$ line. Each of the labeled narrow lines is modeled by a single Gaussian profile. The broad $\mathrm{H} \alpha$ line is represented by the three broader Gaussian profiles. The individual Gaussian components are plotted below the spectrum, together with the residuals (dots) resulting from a simultaneous fit to the spectrum of all the Gaussian profiles plus a fixed continuum level. The three "unmarked" Gaussian profiles are a fit to remove nuisance structure due to the double-peaked profile. [See the electronic edition of the Journal for a color version of this figure.]

erating a "structure map," which is based on the RichardsonLucy image restoration technique (e.g., Pogge \& Martini 2002). The second method that we have applied for enhancing the morphological structures makes use of the IRAF STSDAS/ ELLIPSE routine to construct an elliptical model for the galaxy. The structures are revealed in the residual image obtained by subtracting the model from the original image (see Fig. 3).

The original ACS image shows several dark filaments, in the form of spirals, that are enhanced in the processed images. A comparison with the $J$-band image of PMR05 shows good agreement between the spiral structures seen in the optical and near-infrared, and supports their interpretation that the dark filaments map dust spirals that converge on the unresolved nucleus. The dark filaments form a pattern similar to that seen in the velocity residuals. However, a detailed comparison of the velocity residual map with the ACS image shows that the spiral structure identified in the former is rotated relative to that traced by the dark filaments in the latter. To illustrate this, we have overplotted the loci of the velocity residual spiral arms on the ACS image (Fig. 3). The radial streaming motions in the ionized gas occur between the spiral dust lanes. The latter are indicated by white crosses in Figure 3.

\section{CONCLUSIONS}

We have mapped the gas velocity field in the inner $7^{\prime \prime} \times$ $15^{\prime \prime}$ of NGC 1097 with the GMOS IFU at an unprecedented spatial resolution. These data have enabled us to disentangle circular from noncircular motions in the ionized gas. The dominant motion is disk rotation, on which are superposed velocity residuals that delineate three spiral arms converging onto the unresolved nucleus. We interpret the residual velocities-which reach $\approx 20 \%$ of the rotational velocity amplitude - as evidence of radial inflow at $\approx 50 \mathrm{~km} \mathrm{~s}^{-1}$ from the region interior to the circumnuclear $0.7 \mathrm{kpc}$ ring, toward the nucleus. This is consistent with recent models of nuclear spirals by Maciejewski 

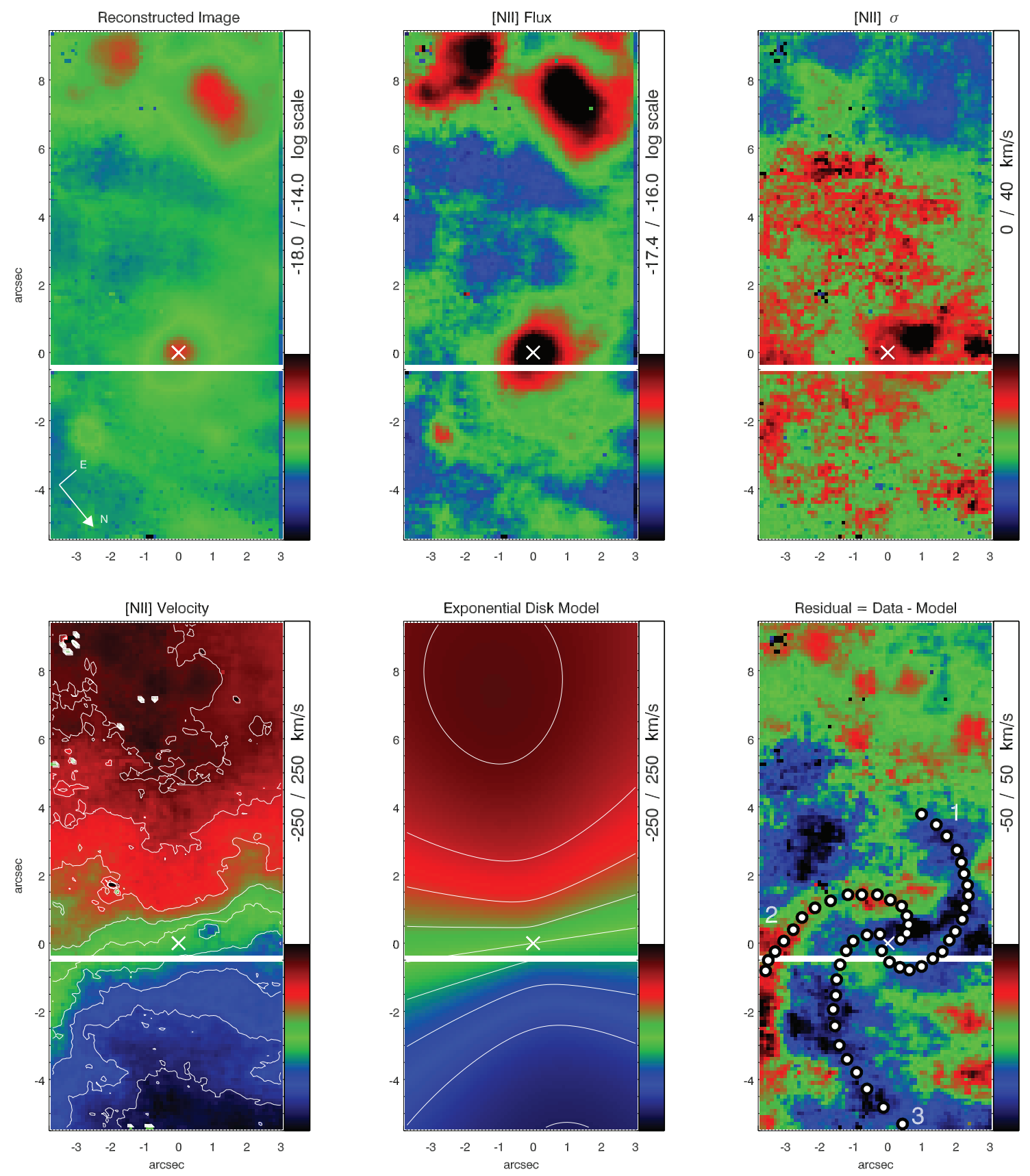

FIG. 2.-GMOS-IFU data results: Reconstructed image formed by collapsing the 6300-6850 $\AA$ wavelength range (top left); [N II] $\lambda 6583$ flux distribution (top middle), together with the kinematic maps derived from the $[\mathrm{N} \mathrm{II]} \mathrm{emission} \mathrm{line,} \mathrm{as} \mathrm{well} \mathrm{as} \mathrm{the} \mathrm{best-fitting} \mathrm{exponential} \mathrm{disk} \mathrm{velocity} \mathrm{field} \mathrm{model} \mathrm{and} \mathrm{residuals.}$ The spiral features are delineated by white dots, with the numbers indicating the different arms as in Fig. 3. In the bottom panels, red color indicates redshift, and blue color indicates blueshift. All panels share the same orientation.

(2004), where such spirals are shocks in gas, capable of feeding the nucleus.

A similar spiral structure delineated by dust lanes is seen in both our optical HST-ACS image and the near-infrared images of PRM05. The radial streaming motions in the ionized gas occur between the spiral dust lanes. On larger scales, similar displacements between gas and dust are common in galactic spiral arms (Tilanus \& Allen 1991). This offset is attributed to the loss of angular momentum of the gas as it passes through the spiral shock (e.g., Combes 1996). Assuming that the dust lanes trace the spiral shock, a similar mechanism may operate in the inner kiloparsecs of NGC 1097. As gas initially on circular orbits passes through the shock, some fraction of its kinetic energy is thermalized, and it loses angular momentum and falls toward the center of the gravitational potential. Taken at face value, the inferred streaming velocities would bring the gas from scales of $10 \mathrm{pc}$ (the spatial resolution of our kinematic maps) down to the nuclear SMBH in approximately 200,000 yr. Such a gas flow could also be responsible for triggering the recently discovered starburst activity in the immediate vicinity of the nucleus (Storchi-Bergmann et al. 2005).

NGC 1097 seems to have all the necessary features to allow gas transfer from galactic scales down to the nucleus (e.g., Englmaier \& Shlosman 2004). It has a large-scale bar, which is most likely the agent to bring gas down to the wellknown circumnuclear ring. It has a secondary bar, which 

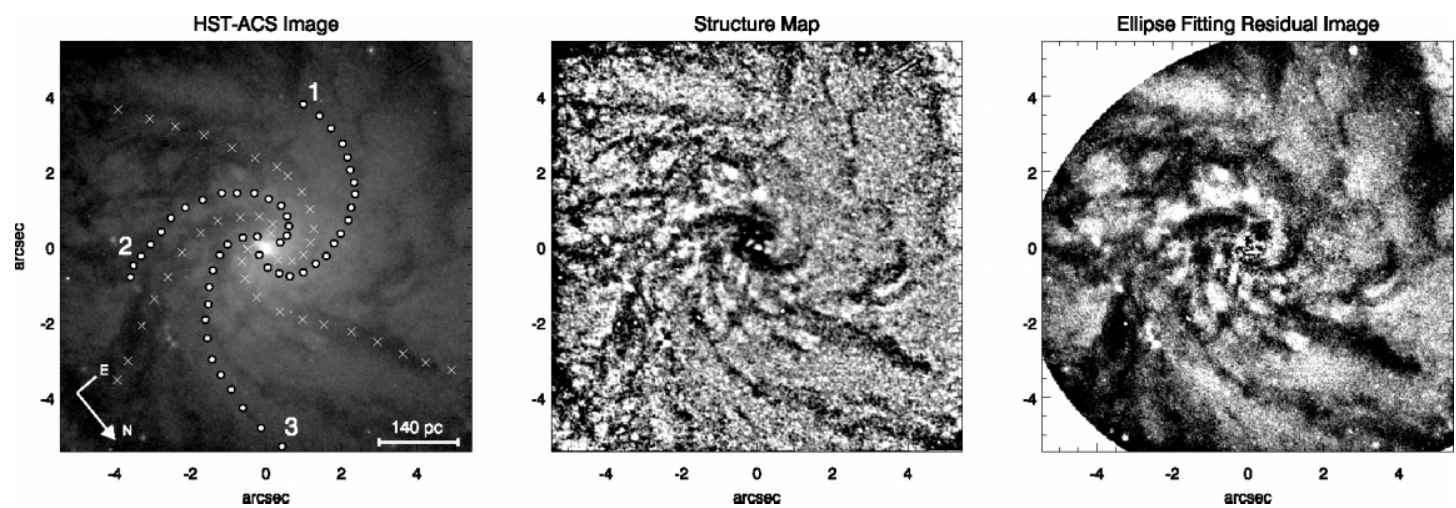

FIG. 3.-Inner $10^{\prime \prime} \times 10^{\prime \prime}$ of the HST-ACS image of NGC 1097 (left), the structure map (middle), and residual between the HST image and the elliptical model $($ right $)$. The angular sampling of 0.025 pixel $^{-1}$ corresponds to $\approx 1.8 \mathrm{pc} \mathrm{pixel}^{-1}$ at the galaxy. The dots delineate the spiral arms found in our kinematic analysis, labeled as in Fig. 2, which follow clockwise rotation (see § 3.1). The white crosses mark the dark regions from Fig. 5 of PMR05.

further funnels gas toward the nuclear region, while here we have mapped streaming velocities along spiral structures, down to at least $10 \mathrm{pc}$ from the nucleus. Furthermore, the high-resolution $H S T$-ACS image suggests that these structures continue down to an $\approx 3.5 \mathrm{pc}$ scale. It is the first time that radial gas inflow has been mapped to such small radii from the central supermassive black hole.

This work is based on observations with the NASA/ESA
HST (HST-GO 09782.01) obtained at the STScI, which is operated by the Association of Universities for Research in Astronomy (AURA), Inc., under NASA contract NAS5-26555. It is also based on data from the Gemini Observatory, which is operated by the AURA, Inc., under a cooperative agreement with the NSF on behalf of the Gemini partnership: the NSF (US), the PPARC (UK), the NRC (Canada), CONICYT (Chile), the ARC (Australia), CNPq (Brazil), and CONICET (Argentina). T. Storchi-Bergmann acknowledges support from $\mathrm{CNPq}$.

\section{REFERENCES}

Combes, F. 1996, in IAU Colloq. 157, Barred Galaxies, ed. R. Buta, D. A. Crocker, \& B. G. Elmegreen (ASP Conf. Ser. 91; San Francisco: ASP), 286 Crenshaw, D. M., Kraemer, S. B., \& Gabel, J. R. 2003, AJ, 126, 1690

Emsellem, E., Fathi, K., Wozniak, H., Ferruit, P., Mundell, C. G., \& Shinnerer, E. 2006, MNRAS, 365, 367

Emsellem, E., Goudfrooij, P., \& Ferruit, P. 2003, MNRAS, 345, 1297

Emsellem, E., Greusard, D., Combes, F., Friedli, D., Leon, S., Pécontal, E., \& Wozniak, H. 2001, A\&A, 368, 52

Englmaier, P., \& Shlosman, I. 2004, ApJ, 617, L115

Fathi, K. 2004, Ph.D. thesis, Univ. Groningen

Fathi, K., et al. 2005, MNRAS, 364, 773

Freeman, K. C. 1970, ApJ, 160, 811

Knapen, J. H. 2005, Ap\&SS, 295, 85

Knapen, J. H., Shlosman, I., Heller, C. H., Rand, R. J., Beckman, J. E., \& Rozas, M. 2000, ApJ, 528, 219

Maciejewski, W. 2004, MNRAS, 354, 892
Maciejewski, W., Teuben, P. J., Sparke, L. S., \& Stone, J. M. 2002, MNRAS, 329,502

Pogge, R. W., \& Martini, P. 2002, ApJ, 569, 624

Prieto, M. A., Maciejewski, W., \& Reunanen, J. 2005, AJ, 130, 1472 (PMR05)

Shaw, M. A., Combes, F., Axon, D. J., \& Wright, G. S. 1993, A\&A, 273, 31

Shlosman, I., Begelman, M. C., \& Frank, J. 1990, Nature, 345, 679

Storchi-Bergmann, T., Baldwin, J. A., \& Wilson, A. S. 1993, ApJ, 410, L11

Storchi-Bergmann, T., Rodriguez-Ardila, A., Schmitt, H. R., Wilson, A. S., \& Baldwin, J. A. 1996, ApJ, 472, 83

Storchi-Bergmann, T., et al. 2003, ApJ, 598, 956 . 2005, ApJ, 624, L13

Tilanus, R. P. J., \& Allen, R. J. 1991, A\&A, 244, 8

Visser, H. C. D. 1980, A\&A, 88, 159

Zurita, A., Relaño, M., Beckman, J. E., \& Knapen, J. H. 2004, A\&A, 413, 73 